\title{
Myelosuppression induced by concurrent chemoradiotherapy as a prognostic factor for patients with locally advanced non-small cell lung cancer
}

\author{
YUKIKO KISHIDA ${ }^{1}$, TAKASHI HIROSE ${ }^{2}$, TAKAO SHIRAI ${ }^{2}$, TOMOHIDE SUGIYAMA ${ }^{2}$, SOUJIRO KUSUMOTO ${ }^{2}$, \\ TOSHIMITSU YAMAOKA ${ }^{2}$, KENTARO OKUDA $^{2}$, MITSURU ADACHI ${ }^{2}$ and AKIHIRO NAKAMURA ${ }^{1}$ \\ ${ }^{1}$ Department of Pharmaceutics; ${ }^{2}$ Division of Respiratory Medicine and Allergology, Department of \\ Internal Medicine, Showa University School of Medicine, Shinagawa, Tokyo 142-8666, Japan
}

Received February 9, 2011; Accepted June 15, 2011

DOI: $10.3892 / \mathrm{ol} .2011 .348$

\begin{abstract}
The aim of the present study was to assess whether myelosuppression during concurrent chemoradiotherapy is a prognostic factor for patients with locally advanced non-small cell lung cancer (NSCLC). We retrospectively analyzed 86 patients with NSCLC who received concurrent platinum-based chemoradiotherapy. Patients were classified into two groups (grades 0-2 and 3-4) according to the most severe neutropenia, anemia or thrombocytopenia observed during concurrent chemoradiotherapy, and survival time and progression-free survival (PFS) time were analyzed. Univariate analysis revealed that overall survival time was significantly longer in patients with grade 0-2 anemia than in those with grade $3-4$ anemia $(p=0.02)$. Survival time did not differ significantly on the basis of the severity of neutropenia or thrombocytopenia. Although pre-treatment white blood cell count was a further prognostic factor in univariate analysis, multivariate analysis revealed that the only independent prognostic factor for overall survival time was anemia. Disease stage was an independent prognostic factor for PFS $(\mathrm{p}=0.04)$, whereas neutropenia, anemia and thrombocytopenia were not. In conclusion, the severity of anemia during concurrent chemoradiotherapy may be a useful prognostic factor in patients with locally advanced NSCLC.
\end{abstract}

\section{Introduction}

Approximately one-third of patients with non-small cell lung cancer (NSCLC) have locally advanced disease at the time of diagnosis. A meta-analysis of randomized trials has

Correspondence to: Dr Takashi Hirose, Division of Respiratory Medicine and Allergology, Department of Internal Medicine, Showa University School of Medicine, 1-5-8 Hatanodai, Shinagawa, Tokyo 142-8666, Japan

E-mail: thirose@med.showa-u.ac.jp

Key words: anemia, chemoradiotherapy, non-small cell lung cancer shown that concurrent chemoradiotherapy improved survival compared to sequential chemoradiotherapy in patients with locally advanced NSCLC (1). Therefore, concurrent chemoradiotherapy has become a standard treatment for patients with locally advanced NSCLC who have a good performance status (PS).

Cytotoxic agents often induce myelosuppression. Certain studies have shown that among patients with breast or ovarian cancer, those with grade 3 or 4 leukopenia or neutropenia during chemotherapy had a longer survival period than patients with grade 0 -2 leukopenia or neutropenia $(2,3)$. Other studies have shown that in patients with advanced NSCLC, small-cell lung cancer (SCLC), gastric or colorectal cancer, survival time is longer when neutropenia is present during chemotherapy than when it is absent (4-9). These reports suggest that neutropenia during chemotherapy is a surrogate marker for antitumor effects, and may indicate that the dosage of chemotherapeutic agents is adequate.

On the other hand, the hypothesis that anemia during chemotherapy is a prognostic factor remains controversial (4,10-13). A study in patients with locally advanced cervical carcinoma found that anemia during chemoradiotherapy was a prognostic factor, whereas another trial found that it was not $(10,11)$. In a study of limited-stage SCLC, anemia during chemoradiotherapy was found not to be a prognostic factor (12). On the other hand, in locally advanced NSCLC, the percentage decline in hemoglobin levels during chemoradiotherapy was reported to be a prognostic factor (13).

Although prognostic factors for patients with locally advanced NSCLC should be clarified, few studies have analyzed these prognostic factors in the disease. Jeremic and Shibamoto have reported that PS, weight loss, disease stage, age and gender were all associated with survival (14). Additionally, the Cancer and Leukemia Group B has reported that pre-treatment anemia and PS have the greatest effects on survival in locally advanced stage III NSCLC (15).

To the best of our knowledge, no studies have evaluated whether the presence of neutropenia during concurrent chemoradiotherapy is a prognostic factor, and few studies have evaluated whether anemia during concurrent chemoradiotherapy is a prognostic factor for patients with locally 
advanced NSCLC undergoing concurrent chemoradiotherapy. Therefore, the association between myelosuppression during concurrent chemoradiotherapy and prognosis in patients with locally advanced NSCLC were examined in this study.

\section{Patients and methods}

Patients. Between May 1999 and December 2008, 118 previously untreated patients with locally advanced stage IIIA or IIIB NSCLC underwent concurrent or sequential chemoradiotherapy at our institution. Of these 118 patients, 86 who had a tumor within an estimated irradiation field no larger than half the hemithorax, and had adequate bone marrow, renal, hepatic and pulmonary function, underwent concurrent chemoradiotherapy with platinum-based doublet chemotherapy. These 86 patients were retrospectively analyzed. This study protocol for retrospective analysis was approved by the ethics committee of the Showa University School of Medicine.

Treatment. Platinum-based doublet chemotherapy was administered every 3 weeks for a maximum of 4 cycles. If stable disease was observed after 2 cycles, the administration of subsequent therapy was left to the discretion of the physician in charge of the patient. A total of 82 patients $(95.3 \%)$ received vinorelbine and cisplatin or carboplatin. Fifty-six patients $(65.1 \%)$ received vinorelbine $\left(20 \mathrm{mg} / \mathrm{m}^{2}\right)$ and cisplatin $\left(40 \mathrm{mg} / \mathrm{m}^{2}\right)$ on days 1 and 8 , and 26 patients $(30.2 \%)$ received vinorelbine $\left(20 \mathrm{mg} / \mathrm{m}^{2}\right)$ and carboplatin [the target area under the concentration vs. time curve (AUC) of $2.5 \mathrm{mg} \mathrm{min} / \mathrm{ml}$ ] on days 1 and 8 . Three patients received cisplatin $\left(80 \mathrm{mg} / \mathrm{m}^{2}\right)$ on day 1 and vindesine $(3 \mathrm{mg}$ / $\mathrm{m}^{2}$ ) on days 1 and 8 , and 1 patient received carboplatin (AUC of $5 \mathrm{mg} \mathrm{min} / \mathrm{ml})$ and docetaxel $\left(60 \mathrm{mg} / \mathrm{m}^{2}\right)$ on day 1 .

If grade 4 leukopenia or neutropenia developed during chemoradiotherapy, granulocyte colony-stimulating factor (G-CSF) was administered according to the guidelines of the Japanese Ministry of Health, Labour and Welfare; radiotherapy was withheld during G-CSF administration. Although the need for transfusion was left to the judgment of the physician, if the platelet count decreased to $<20,000 / \mu 1$, platelets were transfused, and if the hemoglobin level decreased to $<7 \mathrm{~g} / \mathrm{dl}$, erythrocytes were transfused in almost all patients according to the rules of our institution. The doses of all chemotherapy drugs were reduced by $25 \%$ for grade 4 leukopenia or neutropenia lasting 3 days or longer, neutropenic fever during grade 4 neutropenia, or grade 4 thrombocytopenia.

Thoracic radiotherapy consisted of standard chest irradiation in single daily fractions of 2 Gy for 6 weeks, to a total dose of $\sim 60 \mathrm{~Gy}$. The initial dose (until $40 \mathrm{~Gy}$ ) was administered to the original volume that was determined by the size and location of the primary tumor and the draining lymphatic vessels and included a $2-\mathrm{cm}$ margin around the pre-treatment primary tumor and the ipsilateral hilum. The entire width of the mediastinum was included, with a $2-\mathrm{cm}$ margin around the radiographically visible area of involvement. Subsequently, an additional 20 Gy dose was administered to the boost volume, including the entire primary tumor and clinically involved regional hilar and mediastinal lymph nodes, as determined with CT. The original volume was treated with an anterior-posterior parallel-opposed pair of portals, and the boost volume was treated with a pair of oblique fields.
Table I. Patient characteristics.

No. of patients $(\%)$

\begin{tabular}{|c|c|}
\hline \multicolumn{2}{|l|}{ Age (years) } \\
\hline Median (range) & $65(39-79)$ \\
\hline$<70$ & $62(72.1)$ \\
\hline$>70$ & $24(27.9)$ \\
\hline \multicolumn{2}{|l|}{ Gender } \\
\hline Male & $72(83.7)$ \\
\hline Female & $14(16.3)$ \\
\hline \multicolumn{2}{|l|}{ Performance status } \\
\hline 0 & $21(24.4)$ \\
\hline 1 & $62(72.1)$ \\
\hline 2 & $3(3.5)$ \\
\hline \multicolumn{2}{|l|}{ Stage } \\
\hline IIIA & $43(50.0)$ \\
\hline IIIB & $43(50.0)$ \\
\hline \multicolumn{2}{|l|}{ Histological type } \\
\hline Adenocarcinoma & $41(47.7)$ \\
\hline Squamous & $39(45.3)$ \\
\hline Other & $6(7.0)$ \\
\hline \multicolumn{2}{|c|}{ Chemotherapy cycles } \\
\hline 2 & $28(32.6)$ \\
\hline 3 or 4 & $58(67.4)$ \\
\hline \multicolumn{2}{|c|}{ Pre-treatment WBC count } \\
\hline Normal & $59(68.6)$ \\
\hline High & $27(31.4)$ \\
\hline \multicolumn{2}{|c|}{ Pre-treatment hemoglobin } \\
\hline Normal & $61(70.9)$ \\
\hline Low & $25(29.1)$ \\
\hline \multicolumn{2}{|c|}{ Pre-treatment platelet count } \\
\hline Normal & $66(76.7)$ \\
\hline High & $20(23.3)$ \\
\hline \multicolumn{2}{|l|}{ LDH (IU/1) } \\
\hline Normal & $58(67.4)$ \\
\hline High & $28(32.6)$ \\
\hline \multicolumn{2}{|l|}{ Albumin (g/dl) } \\
\hline Normal & $48(55.8)$ \\
\hline Low & $38(44.2)$ \\
\hline
\end{tabular}

Evaluation. Complete blood counts with differential were determined at least once a week during chemoradiotherapy. Patients were divided into 2 groups (those with grade 0-2 and those with grade 3-4) according to the most severe occurrence of neutropenia, anemia or thrombocytopenia during chemoradiotherapy. Other covariables for multivariate analysis were age, gender, PS, histological type, number of cycles of chemotherapy, pre-treatment white blood cell (WBC) count (normal vs. elevated), pre-treatment hemoglobin level (normal vs. low), pre-treatment platelet count (normal vs. elevated), pre-treatment lactate dehydrogenase (LDH) level and pre-treatment albumin level. Normal levels of pre-treatment WBC, hemoglobin, platelets, LDH and albumin were defined as $4,000-9,000 / \mu 1, \geq 12 \mathrm{~g} / \mathrm{dl}, 150,000-400,000 / \mu 1, \leq 220 \mathrm{IU} / 1$ and $\geq 3.8 \mathrm{~g} / \mathrm{dl}$, respectively. 
Table II. Patient characteristics according to the severity of myelosuppression during concurrent chemoradiotherapy.

\begin{tabular}{|c|c|c|c|c|c|c|c|c|c|}
\hline & \multicolumn{3}{|c|}{ Neutropenia } & \multicolumn{3}{|c|}{ Anemia } & \multicolumn{3}{|c|}{ Thrombocytopenia } \\
\hline & Grade $0-2$ & Grade 3-4 & p-value & Grade $0-2$ & Grade 3-4 & p-value & Grade $0-2$ & Grade 3-4 & $\mathrm{p}$-value \\
\hline No. of patients & 9 & 77 & & 51 & 35 & & 83 & 3 & \\
\hline Age & & & 0.22 & & & 0.36 & & & 0.63 \\
\hline$<70$ & 8 & 54 & & 38 & 24 & & 60 & 2 & \\
\hline$>70$ & 1 & 23 & & 13 & 11 & & 23 & 1 & \\
\hline Gender & & & 0.19 & & & 0.31 & & & 0.42 \\
\hline Male & 9 & 63 & & 44 & 28 & & 70 & 2 & \\
\hline Female & 0 & 14 & & 7 & 7 & & 13 & 1 & \\
\hline Performance status & & & 0.30 & & & $<0.01$ & & & 0.57 \\
\hline 0 & 1 & 20 & & 19 & 2 & & 20 & 1 & \\
\hline $1-2$ & 8 & 57 & & 32 & 33 & & 63 & 2 & \\
\hline Stage & & & 0.24 & & & 0.19 & & & 0.50 \\
\hline IIIA & 3 & 40 & & 28 & 15 & & 42 & 1 & \\
\hline IIIB & 6 & 37 & & 23 & 20 & & 41 & 2 & \\
\hline Histological type & & & 0.39 & & & 0.26 & & & 0.13 \\
\hline Adenocarcinoma & 5 & 36 & & 27 & 14 & & 38 & 3 & \\
\hline Squamous & 3 & 36 & & 22 & 17 & & 39 & 0 & \\
\hline $\begin{array}{l}\text { Chemotherapy } \\
\text { cycles }\end{array}$ & & & 0.61 & & & $<0.01$ & & & 0.70 \\
\hline 2 & 3 & 25 & & 26 & 2 & & 27 & 1 & \\
\hline 3 or 4 & 6 & 52 & & 25 & 33 & & 56 & 2 & \\
\hline
\end{tabular}

Tumors were measured prior to treatment and following the second and fourth cycles. Tumor response was assessed using the Response Evaluation Criteria in Solid Tumors version 1.0. Toxicity was assessed using the National Cancer Institute Common Terminology Criteria for Adverse Events version 3.0.

Statistical analysis. Overall survival time was calculated from the start of chemotherapy until death or the last follow-up. Progression-free survival (PFS) was defined as the period from the start of chemotherapy to the identifiable time of the first progression or death from any cause. The Kaplan-Meier method was used to construct survival curves. Survival differences between subgroups were compared by means of the log-rank test. Multivariate analyses were performed with the Cox proportional hazard model. The t-test was used to determine the significance of differences between means. $\mathrm{P}<0.05$ was considered to be statistically significant.

\section{Results}

Patient characteristics. A total of 86 patients who underwent concurrent chemoradiotherapy with platinum-based doublet chemotherapy were eligible for this analysis (Table I). Only 3 patients $(3.5 \%)$ had a PS of 2 . Two patients succumbed due to radiation pneumonitis following the second course of chemotherapy. All of the patients received two cycles of chemotherapy or more. Of the 86 patients, 25 (29.1\%) had anemia prior to treatment: 17 patients $(19.8 \%)$ had hemoglobin levels of $10-12 \mathrm{~g} / \mathrm{dl}$ and 8 patients (9.3\%) had hemoglobin levels of 8-10 g/dl. No significant difference in anemia before chemoradiotherapy between patients with a PS of 1 or 2 and patients with a PS of $0(p=0.54)$. None of the patients received transfusion prior to chemoradiotherapy.

The median follow-up time for all patients was 20 months. At the time of analysis, 22 patients $(25.6 \%)$ were alive and 2 patients $(2.3 \%)$ had been lost to follow-up. Eleven patients $(12.8 \%)$ were free of recurrence and are alive. The disease recurred in 70 patients $(81.4 \%)$. Of these 70 patients, $49(70.0 \%)$ received second-line chemotherapy.

Association between patient characteristics and myelosuppression. Patient characteristics according to the most severe grade of myelosuppression during chemoradiotherapy are shown in Table II. Patients with a PS of 1 or 2 were significantly more likely to develop grade 3 or 4 anemia during chemoradiotherapy than patients with a PS of $0(p<0.01)$. In addition, patients who received 3 or 4 cycles of chemotherapy were significantly more likely to develop grade 3-4 anemia during chemoradiotherapy than patients who received 2 cycles of chemotherapy $(\mathrm{p}<0.01)$. Additionally, patients $(72.1 \%)$ who had anemia before treatment were significantly more likely to have grade 3-4 anemia during chemoradiotherapy than patients $(28.0 \%)$ who did not $(\mathrm{p}<0.01)$. No significant difference was found between patient characteristics and the severity of neutropenia or thrombocytopenia.

All 86 patients had neutropenia during chemoradiotherapy: the most severe neutropenia was grade 1 in 2 patients $(2.3 \%)$, 
Table III. Univariate analysis for overall survival.

\begin{tabular}{|c|c|c|}
\hline & MST (months) & p-value \\
\hline Age (years) & & 0.40 \\
\hline$<70$ & 26 & \\
\hline$>70$ & 20 & \\
\hline Gender & & 0.46 \\
\hline Male & 23 & \\
\hline Female & 32 & \\
\hline Performance status & & 0.11 \\
\hline 0 & 32 & \\
\hline $1-2$ & 20 & \\
\hline Stage & & 0.40 \\
\hline IIIA & 28 & \\
\hline IIIB & 20 & \\
\hline Histological type & & 0.20 \\
\hline Adenocarcinoma & 29 & \\
\hline Squamous & 20 & \\
\hline Chemotherapy cycles & & 0.09 \\
\hline 2 & 20 & \\
\hline 3 or 4 & 32 & \\
\hline Neutropenia (grade) & & 0.44 \\
\hline $0-2$ & 18 & \\
\hline $3-4$ & 23 & \\
\hline Anemia (grade) & & 0.02 \\
\hline $0-2$ & 28 & \\
\hline $3-4$ & 20 & \\
\hline Thrombocytopenia (grade) & & 0.45 \\
\hline $0-2$ & 23 & \\
\hline $3-4$ & 16 & \\
\hline Pre-treatment WBC count & & 0.05 \\
\hline Normal & 26 & \\
\hline High & 19 & \\
\hline Pre-treatment hemoglobin & & 0.76 \\
\hline Normal & 23 & \\
\hline Low & 23 & \\
\hline Pre-treatment platelet count & & 0.22 \\
\hline Normal & 26 & \\
\hline High & 18 & \\
\hline LDH (IU/l) & & 0.75 \\
\hline Normal & 23 & \\
\hline High & 23 & \\
\hline Albumin (g/dl) & & 0.27 \\
\hline Normal & 28 & \\
\hline Low & 20 & \\
\hline
\end{tabular}

grade 2 in 7 patients $(8.1 \%)$, grade 3 in 26 patients $(30.2 \%)$ and grade 4 in 51 patients $(59.3 \%)$. The most severe grade of neutropenia first occurred in the first cycle in 16 patients (18.6\%), in the second cycle in 39 patients $(45.3 \%)$, in the third cycle in 16 patients $(18.6 \%)$ and in the fourth cycle in 15 patients $(17.4 \%)$.

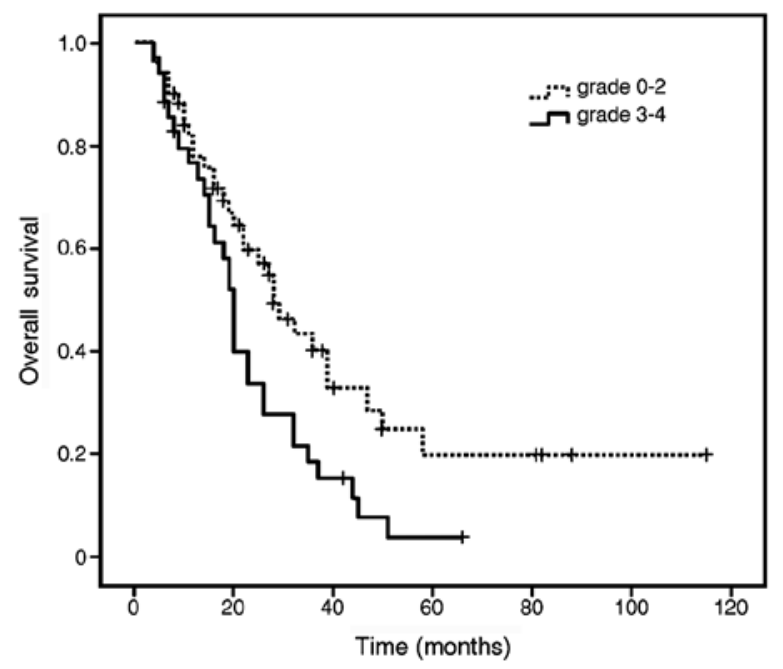

Figure 1. Overall survival time estimated with the Kaplan-Meier method according to the severity of anemia during concurrent chemoradiotherapy. The median survival time was significantly longer in patients with grade $0-2$ anemia during chemoradiotherapy ( 28 months) than in patients with grade 3-4 anemia (20 months; $\mathrm{p}=0.016)$.

G-CSF was administered during 31.9\% of courses (88 of 276 courses; median duration of administration, 2 days; range 1-6).

A total of 84 patients $(97.7 \%)$ had anemia during chemoradiotherapy: the most severe anemia was grade 1 in 9 patients $(10.5 \%)$, grade 2 in 40 patients $(46.5 \%)$, grade 3 in 25 patients $(29.1 \%)$ and grade 4 in 10 patients $(11.6 \%)$. The most severe grade of anemia first occurred in the first cycle in 4 patients $(4.8 \%)$, in the second cycle in 29 patients $(34.5 \%)$, in the third cycle in 14 patients $(16.7 \%)$ and in the fourth cycle in 37 patients $(44.0 \%)$. Fifteen patients received erythrocyte transfusions during chemoradiotherapy.

Forty-seven patients $(54.7 \%)$ had thrombocytopenia during chemoradiotherapy: the most severe thrombocytopenia was grade 1 in 40 patients (46.5\%), grade 2 in 4 patients $(4.7 \%)$, grade 3 in 2 patients $(2.3 \%)$ and grade 4 in 1 patient $(1.2 \%)$. The most severe grade of thrombocytopenia first occurred in the first cycle in 2 patients $(4.3 \%)$, in the second cycle in 23 patients $(48.9 \%)$, in the third cycle in 6 patients $(12.8 \%)$ and in the fourth cycle in 16 patients $(34.0 \%)$. One patient received platelet transfusion during chemoradiotherapy.

Dose intensity. The dosage of chemotherapeutic agents was evaluated in the 82 patients who received a combination of vinorelbine and cisplatin or carboplatin. A total of 177 cycles of cisplatin and vinorelbine were administered, and 90 cycles of carboplatin and vinorelbine were administered. Doses were adjusted in $57.1 \%$ of patients who received cisplatin and vinorelbine, and in $61.5 \%$ of patients who received carboplatin and vinorelbine, mostly due to the occurrence of grade 4 neutropenia lasting $\geq 3$ days, grade 3 neutropenic fever or grade 4 thrombocytopenia. The mean delivered doses as a percentage of the planned doses were $92.1 \%$ for cisplatin, $97.0 \%$ for carboplatin and $92.7 \%$ for vinorelbine.

A total of 83 patients $(96.5 \%)$ completed radiotherapy according to dose and schedule modification. Three patients did not complete radiotherapy due to radiation pneumonitis, severe myelosuppression or general condition deterioration. 
Association between survival and myelosuppression. For all 86 patients, the median survival time (MST) was 23 months, and the median PFS time was 10 months. The MST was significantly longer in patients who had grade 0-2 anemia during chemoradiotherapy (28 months) than in patients who had grade 3-4 anemia (20 months; $p=0.02$; Fig. 1 , Table III). The pre-treatment WBC count was another prognostic factor for survival time in univariate analysis $(\mathrm{p}=0.047)$. Survival time did not correlate with pre-treatment hemoglobin levels. The MST did not differ significantly between patients who had grade 0-2 neutropenia or thrombocytopenia and those who had grade 3-4 neutropenia or thrombocytopenia. Multivariate analysis revealed that the severity of anemia during concurrent chemoradiotherapy was the only independent prognostic factor for survival time $(\mathrm{p}=0.04)$.

The disease stage was the only independent prognostic factor for PFS (Table IV). The PFS time was significantly longer in patients with stage IIIA disease (10 months) than in those with stage IIIB disease (7 months; $\mathrm{p}=0.04$ ). The median PFS time did not differ significantly between patients who had grade 0-2 anemia and those who had grade 3-4 anemia $(p=0.28)$. No significant association was found between PFS time and the severity of neutropenia or thrombocytopenia during chemoradiotherapy.

\section{Discussion}

Few studies have examined whether myelosuppression is a prognostic factor for patients with locally advanced NSCLC who have undergone chemoradiotherapy. Additionally, despite receiving identical chemotherapy regimens and doses, Japanese patients are more likely to experience myelosuppression than patients in Western countries (16). Thus, results may differ between Japanese patients and patients in Western countries. Therefore, we examined the association between myelosuppression during concurrent chemoradiotherapy and prognosis in Japanese patients with locally advanced NSCLC. In the present study, results showed that the severity of neutropenia during concurrent chemoradiotherapy was not a prognostic factor in patients with locally advanced NSCLC. Furthermore, the severity of anemia during chemoradiotherapy was found to be the only prognostic factor in patients with locally advanced NSCLC.

A number of authors have reported that the presence or severity of myelosuppression during chemotherapy was a prognostic factor in patients with breast, ovarian, gastric and colorectal cancer $(2-4,8)$. Moreover, findings of studies in patients with advanced NSCLC have shown that patients with neutropenia receiving chemotherapy survived significantly longer than patients without neutropenia (5-7). Although the dose of numerous anticancer agents is determined on the basis of body surface area, this practice has been criticized (17) due to of interpatient pharmacokinetic variability in metabolism and excretion due to a variety of factors, including pharmacogenetic background (18). The absence of neutropenia during chemotherapy may indicate that the dosage of chemotherapeutic agents is not sufficient to produce their full antitumor effect. It appears that neutropenia may be used as a pharmacodynamic marker to calculate biologically effective doses for dose escalation regimens in individual
Table IV. Univariate analysis for PFS.

\begin{tabular}{|c|c|c|}
\hline & $\begin{array}{l}\text { Median PFS } \\
\text { (months) }\end{array}$ & p-value \\
\hline Age (years) & & 0.13 \\
\hline$<70$ & 10 & \\
\hline$>70$ & 18 & \\
\hline Gender & & 0.97 \\
\hline Male & 10 & \\
\hline Female & 12 & \\
\hline Performance status & & 0.47 \\
\hline 0 & 13 & \\
\hline $1-2$ & 10 & \\
\hline Stage & & 0.04 \\
\hline IIIA & 13 & \\
\hline IIIB & 10 & \\
\hline Histological type & & 0.91 \\
\hline Adenocarcinoma & 11 & \\
\hline Squamous & 10 & \\
\hline Chemotherapy cycles & & 0.68 \\
\hline 2 & 10 & \\
\hline 3 or 4 & 13 & \\
\hline Neutropenia (grade) & & 0.86 \\
\hline $0-2$ & 9 & \\
\hline $3-4$ & 11 & \\
\hline Anemia (grade) & & 0.58 \\
\hline $0-2$ & 11 & \\
\hline $3-4$ & 10 & \\
\hline Thrombocytopenia (grade) & & 0.22 \\
\hline $0-2$ & 11 & \\
\hline $3-4$ & 10 & \\
\hline Pre-treatment WBC count & & 0.28 \\
\hline Normal & 11 & \\
\hline High & 10 & \\
\hline Pre-treatment hemoglobin & & 0.81 \\
\hline Normal & 11 & \\
\hline Low & 10 & \\
\hline Pre-treatment platelet count & & 0.83 \\
\hline Normal & 11 & \\
\hline High & 9 & \\
\hline LDH (IU/l) & & 0.80 \\
\hline Normal & 11 & \\
\hline High & 11 & \\
\hline Albumin (g/dl) & & 0.88 \\
\hline Normal & 12 & \\
\hline High & 10 & \\
\hline
\end{tabular}

patients (19). Subsequently, neutropenia may be used as a dosage-decision factor. On the other hand, in patients with NSCLC, no significant difference in overall survival time has been found between patients with grade 1-2 neutropenia 
during chemotherapy and those with grade 3-4 neutropenia (5-7). In the present study, no significant difference in survival time was found between patients with grade 0-2 neutropenia during chemoradiotherapy and patients with grade $3-4$. We believe that the dosages of chemotherapeutic agents administered to patients in our analysis were sufficient to produce the antitumor effect, since all patients developed neutropenia, and the severity of neutropenia during chemoradiotherapy was not a prognostic factor.

In this study, the MST was significantly longer in patients who had grade 0-2 anemia during chemoradiotherapy than in patients who had grade 3-4 anemia. On the other hand, no significant association was found between survival time and pre-treatment anemia. Whether anemia before or during chemoradiotherapy is a prognostic factor remains controversial. A study involving patients with locally advanced cervical carcinoma found that anemia during chemoradiotherapy, but not pre-treatment anemia, was a prognostic factor, whereas the results of another study found that anemia before chemoradiotherapy, but not during treatment, was a prognostic factor $(10,11)$. In limited-stage SCLC, neither pre-treatment anemia nor anemia during chemoradiotherapy was reported to be a prognostic factor (12). In locally advanced NSCLC, the percentage decline in hemoglobin levels during chemoradiotherapy was reported to be a prognostic factor, whereas the pre-treatment hemoglobin level, the mean hemoglobin level and the minimum hemoglobin level during chemoradiotherapy were reportedly not prognostic factors (13). If presence of anemia during chemoradiotherapy is a prognostic factor, early correction of anemia may be a valuable strategy to improve therapeutic outcomes, quality of life and tolerance of therapy, although transfusion remains the only acceptable treatment for anemia in patients with cancer who are undergoing chemotherapy or chemoradiotherapy, since the use of erythropoietin fails to improve outcomes and increases the risk of thrombotic events (20).

No causes for the association between anemia prior to or during chemoradiotherapy and prognosis for cancer have been definitively established, since the causes of anemia itself are numerous and include blood loss, bone marrow invasion, chronic disease, tumor burden and treatment-related inhibition of erythropoiesis. However, one hypothesis is that anemia increases the severity of tumor hypoxia, leading to poor outcomes (21). Hypoxia causes resistance to radiotherapy and chemotherapy by depriving tumor cells of the oxygen essential for maximal cytotoxic activity. Additionally, hypoxia mediates changes at the transcriptional and the translational level, which affect diverse cellular processes, such as tumor angiogenesis, apoptosis, cellular proliferation and metabolic adaptation (22).

The present study has certain limitations. First, the frequency of blood examination was variable, since our analysis was retrospective. However, complete blood counts with differential were determined at least once a week in all 86 patients and twice a week in certain patients during chemoradiotherapy. Thus, we believe any bias due to the frequency of blood tests is limited. Second, various chemotherapy regimens were included. However, we only included patients who underwent platinum-based doublet chemotherapy, whereas certain previous reports included different regimens of numbers of chemotherapy drugs $(5,6,13)$. Therefore, the bias of different chemotherapeutic regimens is limited. Third, the use of G-CSF and platelet or transfusion erythrocyte was variable. However, G-CSF was administered only in patients with grade 4 leukopenia or neutropenia and was not administered prophylactically. In addition, according to the rules of our institution, platelets were usually transfused if the platelet count decreased to less than $20,000 / \mu 1$, and erythrocytes were usually transfused if the hemoglobin level decreased to less than $7 \mathrm{~g} / \mathrm{dl}$. Therefore, these results should not be biased by the use of G-CSF or by platelet or erythrocyte transfusion.

In conclusion, the severity of neutropenia during concurrent chemoradiotherapy is not considered to be a prognostic factor in patients with locally advanced NSCLC. On the other hand, the severity of anemia during concurrent chemoradiotherapy may be a useful prognostic factor in patients with locally advanced NSCLC. In the future, large, well-designed prospective trials are required to evaluate the merits of correction of anemia with transfusion during chemoradiotherapy in patients with locally advanced NSCLC.

\section{References}

1. Auperin A, Le Pechoux C, Rolland E, et al: Meta-analysis of concomitant versus sequential radiochemotherapy in locally advanced non-small-cell lung cancer. J Clin Oncol 28: 2692-2699, 2010.

2. Rocconi RP, Matthews KS, Kemper MK, Hoskins KE and Barnes MN: Chemotherapy-related myelosuppression as a marker of survival in epithelial ovarian cancer patients. Gynecol Oncol 108: 336-341, 2008.

3. Saarto T, Blomqvist C, Rissanen P, Auvinen A and Elomaa I: Haematological toxicity: a marker of adjuvant chemotherapy efficacy in stage II and III breast cancer. Br J Cancer 75: 301-305, 1997.

4. Yamanaka T, Matsumoto S, Teramukai S, Ishiwata R, Nagai Y and Fukushima M: Predictive value of chemotherapy-induced neutropenia for the efficacy of oral fluoropyrimidine S-1 in advanced gastric carcinoma. Br J Cancer 97: 37-42, 2007.

5. Di Maio M, Gridelli C, Gallo C, et al: Chemotherapy-induced neutropenia and treatment efficacy in advanced non-small cell lung cancer: a pooled analysis of three randomized trials. Lancet Oncol 6: 669-677, 2005

6. Kishida Y, Kawahara M, Teramukai S, et al: Chemotherapyinduced neutropenia as a prognostic factor in advanced non-small-cell lung cancer: results from Japan Multinational Trial Organization LC00-03. Br J Cancer 101: 1537-1542, 2009.

7. Pallis AG, Agelaki S, Kakolyris S, et al: Chemotherapy-induced neutropenia as a prognostic factor in patients with advanced non-small cell lung cancer treated with front-line docetaxelgemcitabine chemotherapy. Lung Cancer 62: 356-363, 2008.

8. Shitara K, Matsuo K, Takahari D, et al: Neutropenia as a prognostic factor in metastatic colorectal cancer patients undergoing chemotherapy with first-line FOLFOX. Eur J Cancer 45: 1757-1763, 2009.

9. Banerji U, Ashley S, Coward J, et al: The association of chemotherapy induced neutropenia on treatment outcomes in small cell lung cancer. Lung Cancer 54: 371-377, 2006.

10. Ferrandina G, Distefano M, Smaniotto D, et al: Anemia in patients with locally advanced cervical carcinoma administered preoperative radiochemotherapy: association with pathological response to treatment and clinical outcome. Gynecol Oncol 103: 500-505, 2006.

11. Obermair A, Cheuk R, Horwood K, et al: Impact of hemoglobin levels before and during concurrent chemoradiotherapy on the response of treatment in patients with cervical carcinoma. Cancer 92: 903-908, 2001.

12. Laurie SA, Ding K, Whitehead M, et al: The impact of anemia on outcome of chemoradiation for limited small-cell lung cancer: a combined analysis of studies of the National Cancer Institute of Canada Clinical Trials Group. Ann Oncol 18: 1051-1055, 2007.

13. MacRae R, Shyr Y, Johnson D and Choy H: Declining hemoglobin during chemoradiotherapy for locally advanced non-small cell lung cancer is significant. Radiother Oncol 64: 37-40, 2002. 
14. Jeremic B and Shibamoto Y: Pre-treatment prognostic factors in patients with stage III non-small cell lung cancer treated with hyperfractionated radiation therapy with or without concurrent chemotherapy. Lung Cancer 13: 21-30, 1995.

15. Socinski MA, Zhang C, Herndon JE II, et al: Combined modality trials of the Cancer and Leukemia Group B in stage III non-small-cell lung cancer: analysis of factors influencing survival and toxicity. Ann Oncol 15: 1033-1041, 2004.

16. Gandara DR, Kawaguchi T, Crowley J, et al: Japanese-US common-arm analysis of paclitaxel plus carboplatin in advanced non-small-cell lung cancer: a model for assessing population-related pharmacogenomics. J Clin Oncol 27: 3540-3546, 2009.

17. Gurney H: How to calculate the dose of chemotherapy. Br J Cancer 86: 1297-1302, 2002.

18. Evans WE and Mcleod HL: Pharmacogenomics-drug disposition, drug targets, and side effects. N Eng J Med 348: 538-549, 2003
19. Jordan SD, Poole CJ, Archer VR, Steven NM and Burton A: A retrospective evaluation of the feasibility of intrapatient dose escalation as appropriate methodology for dose-ranging studies for combination cytotoxic regimens. Cancer Chemother Pharmacol 52: 113-118, 2003.

20. Pirker R, Ramlau RA, Schuette W, et al: Safety and efficacy of darbepoetin alfa in previously untreated extensive-stage small-cell lung cancer treated with platinum plus etoposide. J Clin Oncol 26: 2342-2349, 2008.

21. Harrison L and Blackwell K: Hypoxia and anemia: factors in decreased sensitivity to radiation therapy and chemotherapy? The Oncologist 9: S31-S40, 2004.

22. Dachs GU and Tozer GM: Hypoxia modulated gene expression: angiogenesis, metastasis and therapeutic exploitation. Eur J Cancer 36: 1649-1660, 2000 